\title{
Manifestações clínicas de pacientes portadores de doença hepática gordurosa não alcoólica
}

Clinical manifestations of patients with non-alcoholic fatty liver disease

\author{
E. I. G. Silva ${ }^{1 *}$; B. E. S. Cunha ${ }^{2}$; S. E. M. Guedes ${ }^{2}$; M. T. O. Tomiya ${ }^{1}$; A. M. D. \\ Silva $^{1}$; C. A. Brito ${ }^{1}$ \\ ${ }^{1}$ Departamento de Nutrição, Instituto de Medicina Integral Professor Fernando Figueira,50070-550, Recife- \\ Pernambuco, Brasil \\ ${ }^{2}$ Deparatmento de Nutrição, Faculdade Pernambucana de Saúde,51180-001, Recife-Pernambuco, Brasil \\ *emerson.iago@hotmail.com
}

(Recebido em 15 de setembro de 2018; aceito em 08 de outubro de 2018)

\begin{abstract}
Este estudo buscou verificar as principais manifestações clínicas apresentadas por pacientes portadores de doença hepática gordurosa não alcoólica (DHGNA) com diferentes graus de acúmulo esteatogênico acompanhados em dois centros ambulatoriais de Recife-PE. Estudo transversal, multicêntrico, de natureza quantitativa, realizado com indivíduos adultos diagnosticados com DHGNA via ultrassonografia. As variáveis coletadas foram: idade, gênero, grau de DHGNA, dados bioquímicos (TGO, TGP, GGT, FA, colesterol total, HDL, LDL, triglicerídeos Glicemia em jejum,), pressão arterial e dados antropométricos (peso, altura, circunferência da cintura e índice de massa corporal). Foram revisados os prontuários e elencados as manifestações clinicas apresentadas. Para definir síndrome metabólica utilizou-se os critérios do NCEP-ATPIII e para definir obesidade os critérios da OMS e OPAS. As variáveis foram analisadas como frequências e associações por teste de inferência estatística. Foram selecionados 110 indivíduos, em sua maioria do sexo feminino $(80,9 \%)$ e com menos de 60 anos. O grau de esteatose moderado foi prevalente na amostra. Das manifestações encontradas, a hipertensão arterial $(80 \%)$, obesidade $(73,6 \%)$ e síndrome metabólica $(67,3 \%)$ foram as mais importantes. As médias de glicemia em jejum se associaram aos graus de esteatose $(\mathrm{p}=0.023)$, assim como o colesterol total $(\mathrm{p}=0.038)$. Inúmeras manifestações clinicas acham-se no escopo da DHGNA. Nesse estudo, a hipertensão arterial e obesidade foram as mais importantes. Ademais, um descontrole glicêmico tanto quanto maior aumenta com a gravidade da doença e, portanto, piora o prognóstico. Logo, evidencia-se a necessidade ações multidisciplinares promotoras de saúde ligadas a mudanças no estilo de vida.

Palavras-chave: hepatopatia, doenças crônicas, obesidade
\end{abstract}

To verify the main clinical manifestations presented by patients with non-alcoholic fatty liver disease (NAFLD) with different degrees of steatogenic accumulation followed at two outpatient centers in RecifePE. Cross-sectional, multicenter, quantitative study of adult individuals diagnosed with NAFLD by ultrasonography. The variables were: age, gender, degree of NAFLD, biochemical data (TGO, TGP, GGT, FA, total cholesterol, HDL, LDL, fasting glycemia triglycerides), blood pressure and anthropometric data (weight, height, circumference waist and body mass index). We reviewed the medical records and listed the clinical manifestations presented. To define metabolic syndrome, the NCEP-ATPIII criteria were used, and WHO and OPAS criteria were defined as obesity. The variables were analyzed as frequencies and associations by statistical inference test. A total of 110 individuals, mostly female (80.9\%) and less than 60 years old, were selected. The degree of moderate steatosis was prevalent in the sample. Of the manifestations found, arterial hypertension $(80 \%)$, obesity $(73.6 \%)$ and metabolic syndrome $(67.3 \%)$ were the most important. Fasting blood glucose levels were associated with steatosis degrees $(\mathrm{p}=0.023)$, as well as total cholesterol $(\mathrm{p}=0.038)$. Numerous clinical manifestations are within the scope of NAFLD. In this study, arterial hypertension and obesity were the most important. In addition, a glycemic uncontrolled as much increases with the severity of the disease and, therefore, worsens the prognosis. Therefore, there is a need for multidisciplinary health promotion actions linked to changes in lifestyle.

Keywords: liver disease, chronic diseases, obesity 


\section{INTRODUÇÃO}

A Doença hepática gordurosa não alcoólica (DHGNA) é um termo clínico que indica a deposição e acúmulo de gordura no fígado superior a 5\% do peso do fígado com efeitos hepatotóxicos. Esse distúrbio apresenta um amplo espectro histológico, desde a simples esteatose hepática a uma condição progressiva de lesão hepática, chamada esteato-hepatite não alcoólica [1]. Com o aparecimento da epidemia de obesidade por consequência do consumo de dietas calóricas e sedentarismo, a DHGNA está se tornando um grave problema de saúde global, representando a principal causa de doença hepática crônica no mundo [2,3]. Antecipa-se que o ônus dessa doença não transmissível (DHGNA) e de outras excedam o das doenças transmissíveis. As projeções, por exemplo, revelam que até 2020, as verbas em saúde das doenças transmissíveis sejam elevadas em 20\% e das doenças não transmissíveis aumentem em 60\% [4], no que se refere a terapias medicamentosas e custos hospitalares com internamentos $\mathrm{e}$ complicações.

Embora sua taxa de progressão seja mais lenta que outros tipos de doença hepáticas [5], a pandemia de DHGNA aumenta de forma paralela com as epidemias de obesidade, diabetes tipo 2 (DM2) e síndrome metabólica (SM) [6]. Nas populações da Europa e dos Estados Unidos (EUA) sua prevalência alcança de 25\%-30, mas estes números elevam-se a 80\%-90\% em coortes selecionados de sujeitos dismetabólicos [4,7,8]. Contudo, existem áreas do mundo, como a América do Sul e o Oriente Médio, em que a prevalência média atinge > 30\%. Por outro lado, a África tem a menor prevalência de DHGNA em todo o mundo [8].

Os pacientes com DHGNA apresentam maior risco de doença cardiovascular prematura, bem como de mortalidade relacionada ao fígado. Isso porque em até 50\% dos casos, a DHGNA pode evoluir para o hepatocarcinoma celular (HCC) na ausência de cirrose, uma circunstância que frequentemente piora o resultado. Além disso, co-morbidades freqüentes da DHGNA incluem condições específicas de cardio-renal-metabólicas e aumento do risco de câncer hepático / extrahepático [9].

Ao contrário de outras doenças hepáticas crônicas, a DHGNA geralmente está associada à resistência à insulina e, muitas vezes, a pelo menos uma característica da síndrome metabólica (SM): obesidade, glicemia de jejum aumentada, hipertrigliceridemia, colesterol de baixa densidade e hipertensão [5]. Aceita-se que a DHGNA é uma doença heterogênea com uma variedade de vias patogênicas, contudo as manifestações e/ou condições clinicas são variáveis, razão de realizar levantamentos clinico-patológicos de tais eventos, uma vez que predizem as condições de morbimortalidade dos indivíduos e as metas de tratamento, além de esclarecer ou indiciar as rotas dismetabólicas apresentáveis.

Diante das exposições realizadas, a proposta deste estudo foi verificar as principais manifestações clínicas apresentadas por pacientes portadores de doença hepática gordurosa não alcoólica com diferentes graus de acúmulo esteatogênico atendidos em centros ambulatoriais de Recife-Pernambuco, Brasil.

\section{METODOLOGIA}

Esse é um estudo de delineamento transversal, multicêntrico, com abordagem quantitativa, sendo realizado nos ambulatórios de nutrição do Instituto de Medicina Integral Professor Fernando Figueira e Instituto do Fígado de Pernambuco - PE, ambos, centros de atendimento à população local e áreas afins, localizados em Recife-PE, Brasil. A população elegível para o estudo foi formada por pacientes em acompanhamento ambulatorial das referidas Instituições de ambos os sexos, acima de 18 anos, com diagnóstico de DHGNA via ultrassonografia (USG). A amostragem foi não probabilística e o período de execução da pesquisa transcorreu de abril a novembro de 2017 com pacientes atendidos nesse período. Essa pesquisa constitui um recorte de um projeto maior intitulado: "Parâmetros clínicos e nutricionais de pacientes portadores de esteatose hepática não alcoólica e sua relação com a síndrome metabólica" aprovado pelo comitê de ética e pesquisa do Instituto de Medicina Integral Professor Fernando Figueira, sob o número 64898517.1.0000.5201. Todos os participantes foram avisados 
e esclarecidos da natureza e perspectivas do projeto. A participação dos indivíduos ocorreu mediante assinatura do Termo de Compromisso Livre e Esclarecido (TCLE).

Todos os pacientes com diagnóstico de DHGNA via USG, com mais de 18 anos foram convidados a participar do estudo. Indivíduos com idade inferior a 18 anos; etilista ativo; gestantes; sujeitos em uso de medicação esteatogênica ou hepatotóxica; diagnóstico de alguma desordem hepática hereditária ou soropositividade para vírus da hepatite B (HVB) e C (HVC) foram excluídos da pesquisa. Pacientes com alguma doença incapacitante (reumáticas, neuromusculares, osteoarticulares ou degenerativas) que se inviabilizava antropometria e colhimento de informações também foram exclusos. A validação das informações ocorreu mediante inspeção em prontuários médicos.

Para coleta dos dados, os prontuários dos pacientes foram acessados e revisados. Dados como idade, sexo e manifestações clínicas foram notificados nos prontuários para garantia de veracidade informacional. Quanto as variáveis bioquímicas, os pacientes foram submetidos a exames sanguíneos no ato da entrevista. As variáveis laboratoriais de interesse coletadas foram: transaminases hepáticas: glutâmico-oxaloacetica (TGO) e glutâmico - pirúvica (TGP); glutamiltransferase (GGT); fosfatase alcalina (FA), colesterol total (CT) e suas frações (High Density Lipoprotein - HDL; Low Density Lipoprotein - LDL); triglicerídeos (TG), glicemia em jejum (GJ). O preparo de jejum mínimo de $8 \mathrm{~h}$, de sólidos e líquidos foi estabelecido para todos os indivíduos. A pressão arterial foi aferida em todos os pacientes, seguindo as recomendações das VI Diretrizes Brasileiras de Hipertensão [9]. Para classificar os indivíduos como obesos, foram coletados dados de peso, altura, circunferência da cintura e índice de massa corporal, cujos valores foram avaliados segundos os critérios da Organização Mundial de Saúde - OMS (2000)[10] para adultos e os critérios da Organização Pan-americana de Saúde - OPAS (2002)[11], para idosos.

A presença de Síndrome metabólica na população obedeceu aos critérios do National Cholesterol Education Program's Adult Treatment Panel III (NECP - ATP III) (2001), que caracteriza SM na presença de 3 ou mais de cinco critérios: 1) obesidade central definida pela circunferência da cintura (CC): $\geq 102$ para homens e $\geq 88$ para mulheres; 2$)$ lipoproteína de alta densidade - colesterol (HDLc): $<50 \mathrm{mg} / \mathrm{dL}(<1.0 \mathrm{mmol} / \mathrm{L})$ para mulheres ou $<40 \mathrm{mg} / \mathrm{dL}(0.9$ $\mathrm{mmol} / \mathrm{L}$ ) para homens; 3) triglicerídeos $\geq 150 \mathrm{mg} / \mathrm{dL}$ (1.7 mmol/L); 4) pressão artéria diastólica (PAS): > $130 \mathrm{mmHg}$ ou pressão arterial diastólica (PAD) $\geq 85 \mathrm{mmHg}$; 5) glicose plasmática em jejum $110 \mathrm{mg} / \mathrm{dL}$ (6.1 mmol/L). Creditou-se como critério abrangido, independente da aferição da pressão arterial (PA), indivíduos com diagnóstico médico prévio de hipertensão arterial sistêmica (HAS) e/ou em uso de anti-hipertensivo. A semelhança, atribuímos como critério incluso àqueles com diagnóstico de diabetes.

Todos os dados foram compilados no Microsoft Excel 2013. Para análise estatística os dados foram lançados no programa SPSS versão 13.0(SPSS Inc., Chicago, IL, USA). As variáveis contínuas foram testadas quanto à normalidade da distribuição, pelo teste de KolmogorovSmirnov, as que apresentaram distribuição normal foram descritas na forma de média e desvio padrão, e as com distribuição não normal, em mediana e intervalo interquartílico. Na descrição das proporções, a distribuição binomial foi aproximada à distribuição normal, pelo intervalo de confiança de $95 \%$. Nos testes de inferência estatística, as proporções foram comparadas pelo teste do Qui quadrado de Pearson. Na comparação entre as médias foi utilizada a análise de variância (ANOVA uma via), quando os critérios de homocedasticidade e distribuição normal foram atingidos, e o teste de Tukey a posteriori. Os testes de Kruskal Wallis e "U" Mann Whitney foram utilizados quando os critérios de normalidade e/ou homocedasticidade não foram atingidos. Foi utilizado o nível de significância de 5,0\% para rejeição de hipótese de nulidade.

\section{RESULTADOS}

Participaram desse estudo 110 indivíduos com doença hepática gordurosa não alcoólica via USG que respeitaram os critérios de elegibilidade. Destes, $80,9 \%(\mathrm{n}=89)$ pertenciam ao sexo feminino, enquanto $19,1 \%(\mathrm{n}=21)$ ao sexo masculino. A idade média foi 55,4 $(\mathrm{DP} \pm 11,1)$ no sexo 
feminino e 52,8 $(\mathrm{DP} \pm 13,4)$ para homens. A maior parte dos indivíduos da amostra eram adultos, ou seja, com menos de 60 anos, e um percentual 63,6\% $(n=70)$ representou.

Quanto á gravidade de acúmulo esteatogênico, a maioria dos indivíduos apresentava um grau intermediário $(45,5 \% ; n=50)$, seguido por leve $(42,7 \% ; n=47)$ e grave $(11,8 \% ; n=13)$.

A tabela 1 traz o perfil das manifestações clínicas apresentadas pelos participantes da pesquisa. Como pode ser observado, a hipertensão arterial (80\%), seguida de obesidade $(73,6 \%)$ e síndrome metabólica $(67,3 \%)$ foram as manifestações mais prevalentes. O diabetes mellitus $(32,7 \%)$ foi o menos frequente.

Tabela 1 - Perfil clínico de manifestações clinico-patológicas apresentadas por pacientes com esteatose hepática não alcoólica. Recife, Pernambuco, Brasil, 2018.

\begin{tabular}{lll}
\hline Manifestação clínicas & N & \% \\
\hline Síndrome Metabólica & 74 & 67,3 \\
Hipertensão arterial & 88 & 80,0 \\
Diabetes Mellitus & 36 & 32,7 \\
Obesidade & 81 & 73,6 \\
Hipertrigliceridemia & 37 & 33,6 \\
Hipercolesterolemia & 45 & 40,9 \\
HDL baixo & 60 & 54,5 \\
LDL alto & 45 & 40,9 \\
\hline
\end{tabular}

Na tabela 2 podem ser observadas as variáveis clínicas e bioquímicas coletadas de acordo com os diferentes graus de esteatose. Das variáveis de componente lipídico apenas o colesterol total foi estatisticamente significante $(\mathrm{p}<0,038)$, em relação ao grau mais severo da doença, não sendo observado nos graus leve e intermediário. Os pacientes com maior gravidade apresentaram valores menores de colesterol total sérico.

Tabela 2 - Comparação entre variáveis clínicas e bioquímicas com o grau de esteatose em pacientes. Recife, Pernambuco, Brasil, 2018

\begin{tabular}{ccccc}
\hline Variáveis & \multicolumn{4}{c}{ DGHNA } \\
\cline { 2 - 4 } & Leve & Moderada & Grave & \\
\cline { 2 - 4 } & Média \pm DP & Média \pm DP & Media \pm DP & $\mathrm{p}^{*}$ \\
CT & $192,1 \pm 48,2^{\mathrm{a}}$ & $197,4 \pm 41,2^{\mathrm{a}}$ & $161,5 \pm 43,7^{\mathrm{b}}$ & $\mathbf{0 , 0 3 8}$ \\
LDL & $113,9 \pm 48,7$ & $116,0 \pm 38,7$ & $88,24 \pm 35,3$ & 0,110 \\
& Mediana (IQ) & Mediana (IQ) & Mediana (IQ) & $\mathrm{p}^{\#}$ \\
PAD & $80(80-90)$ & $80(80-90)$ & $80(80-95)$ & 0,990 \\
PAS & $130(120-140)$ & $130(120-140)$ & $130(120-151)$ & 0,569 \\
TGO & $22,0(17,0-42,0)$ & $24,0(20,0-35,4)$ & $20,0(17,0-29,5)$ & 0,300 \\
TGP & $27,0(20,0-42,0)$ & $29,0(19,1-42,0)$ & $25,2(18,5-32,5)$ & 0,653 \\
GGT & $37,98(22,0-58,0)$ & $52,5(29,9-88,0)$ & $37,0(21,5-103,2)$ & 0,088 \\
FA & $78,6(65,0-96,0)$ & $76,0(62,1-92,1)$ & $76,9(52,3-92,1)$ & 0,448 \\
GJ & $95,4(86,0-105,9)^{\mathrm{x}}$ & $107,0(88,7-137,1)^{\mathrm{y}}$ & $140,9(93,8-162,5)^{\mathrm{yz}}$ & $\mathbf{0 , 0 2 3}$ \\
HDL & $45,0(37,7-51,0)$ & $46,2(42,7-53,0)$ & $47,9(40,5-59,3)$ & 0,477 \\
TG & $138,0(103-168)$ & $129,0(106,5-166,7)$ & $140,0(101,39-168,9)$ & 0,903 \\
\hline
\end{tabular}

*Teste ANOVA; ${ }^{*}$ Teste Kruskal-Wallis; Letras diferentes significa diferença estatística, $\mathrm{p}<0,05$ ( ${ }^{\text {abc }}$ pos hoc de Tukey, xyzTeste Mann-Whitney); DP: Desvio Padrão; IQ: Intervalo Interquartílico. DHGNA - Doença Hepática Gordurosa não alcoólica; CT - Colesterol total; LDL - Low Density Lipoprotein; PAD - Pressão arterial diastólica; PAS - Pressão arterial sistólica; TGO - Glutâmico-oxaloacetica; TGP - Glutâmico - pirúvica; GGT - Glutamiltransferase; FA Fosfatase alcalina; GJ - Glicemia em jejum; HDL - High Density Lipoprotein; TG -Triglicerídeos. 
Além do colesterol, a glicemia em jejum também foi estatisticamente significante na população ( $\mathrm{p}<0.023)$, aumentando ao nível que a infiltração gordurosa avança.

$\mathrm{Na}$ tabela 3, verifica-se as principais manifestações clínicas apresentadas pelos pacientes e sua associação com o grau de esteatose hepática. Não foi observada nenhuma manifestação clínica se associando proporcionalmente aos diferentes graus de esteatose hepática, embora o Diabetes Mellitus tenha tendenciado ( $p>0,06$ ).

Tabela 3 - Associação entre manifestações clínicas com o grau de esteatose em pacientes. Recife, Pernambuco, Brasil,2018.

\begin{tabular}{|c|c|c|c|c|c|c|c|}
\hline & \multicolumn{6}{|c|}{ DGHNA } & \multirow{3}{*}{$\mathbf{p}^{\mathbf{a}}$} \\
\hline & \multicolumn{2}{|c|}{ Leve } & \multicolumn{2}{|c|}{ Moderada } & \multicolumn{2}{|c|}{ Grave } & \\
\hline & $\mathrm{n}$ & $\%$ & $\mathrm{n}$ & $\%$ & $\mathrm{n}$ & $\%$ & \\
\hline \multicolumn{8}{|c|}{ Síndrome Metabólica } \\
\hline Sim & 27 & 36,5 & 36 & 48,6 & 11 & 14,9 & 0,114 \\
\hline Não & 20 & 55,6 & 14 & 38,9 & 2 & 5,6 & \\
\hline \multicolumn{8}{|c|}{ Hipertensão Arterial } \\
\hline Sim & 34 & 38,6 & 43 & 48,9 & 11 & 12,5 & 0,243 \\
\hline Não & 13 & 59,1 & 7 & 31,8 & 2 & 9,1 & \\
\hline \multicolumn{8}{|c|}{ Diabetes Mellitus } \\
\hline Sim & 10 & 27,8 & 22 & 61,1 & 4 & 11,1 & 0,060 \\
\hline Não & 37 & 50,0 & 28 & 37,8 & 9 & 12,2 & \\
\hline \multicolumn{8}{|c|}{ Obesidade } \\
\hline Sim & 31 & 38,3 & 38 & 46,9 & 12 & 14,8 & 0,149 \\
\hline Não & 16 & 55,2 & 12 & 41,4 & 1 & 3,4 & \\
\hline \multicolumn{8}{|c|}{ Hipertrigliceridemia } \\
\hline Sim & 11 & 29,7 & 21 & 56,8 & 5 & 13,5 & 0,147 \\
\hline Não & 36 & 49,3 & 29 & 39,7 & 8 & 11,0 & \\
\hline \multicolumn{8}{|c|}{ Hipercolesterolemia } \\
\hline Sim & 19 & 42,2 & 24 & 53,3 & 2 & 4,4 & 0,112 \\
\hline Não & 28 & 43,1 & 26 & 40,0 & 11 & 16,9 & \\
\hline \multicolumn{8}{|c|}{ HDL baixo } \\
\hline Sim & 26 & 43,3 & 26 & 43,3 & 8 & 13,3 & 0,862 \\
\hline Não & 21 & 42,0 & 24 & 48,0 & 5 & 10,0 & \\
\hline \multicolumn{8}{|c|}{ LDL alto } \\
\hline Sim & 21 & 46,7 & 22 & 48,9 & 2 & 4,4 & 0,152 \\
\hline Não & 26 & 40,0 & 28 & 43,1 & 11 & 16,9 & \\
\hline
\end{tabular}

DHGNA - Doença hepática gordurosa não alcoólica; Teste qui-quadrado.

\section{DISCUSSÃO}

A DHGNA é uma condição metabólica complexa e tem sido cada vez mais reconhecida como uma das principais causas de morbidade e mortalidade relacionada ao fígado, tanto com fatores de risco genéticos quanto de estilo de vida [1]. Essa capacidade da DHGNA de elevar os índices de morbimortalidade deve-se, sobretudo, ao fato de associar condições clínicas, exacerbando-as ou convergindo para o seu aparecimento, razão de investir em pesquisas que elucidem suas manifestações.

Segundo Almeida-Valdez (2009) [12] cerca de 90,0\% dos indivíduos com DGHNA têm pelo menos um recurso da síndrome metabólica. Todavia, todos os recursos da SM já foram observados em 33\% dos portadores de DHGNA [12]. Além disso, se a doença hepática gordurosa não alcoólica e a SM coexistem, a regressão da doença é menos provável [13].Como foi observado 
nesse estudo, todos os critérios da síndrome metabólica estavam presentes nos pacientes, sendo a obesidade e a hipertensão os mais relevantes.

Embora tenha sido sugerido que a DHGNA é uma característica hepática da Síndrome Metabólica, um estudo com base na análise de dados de 3846 indivíduos da terceira pesquisa nacional de exames de saúde e nutrição dos Estados Unidos revelou que a DHGNA não é um componente ou manifestação independente de SM, mas sim uma condição fortemente associada aos recursos da síndrome metabólica [14]. Os achados dessa pesquisa mostraram uma prevalência de Síndrome metabólica importante (67,3\%).

A obesidade pode ser considerada uma condição inflamatória crônica de baixo grau e citocinas relacionadas à obesidade, como interleucina-6 (IL-6), adiponectina, leptina e fator de necrose tumoral (TNF) a podem desempenhar papéis importantes no desenvolvimento da DHGNA [5]. Seja no cenário nacional ou internacional, os estudos concordam com o papel imponente da obesidade na etiopatogênese da DHGNA.

Um estudo brasileiro [15] objetivando verificar a relação da DHGNA com componentes da SM e resistência à insulina encontrou uma proporção total de obesos ou com sobrepeso em seu estudo. No campo internacional, um estudo libanês verificou uma prevalência de 56,6\% de indivíduos obesos com DHGNA [16].Igualmente, dados de um trabalho realizado na China também observaram altas proporções de sobrepeso e obesidade, com números de 59\% e 49,4\%, respectivamente [17].Esses dados, embora, inferiores ao nosso estudo sinalizam a obesidade como importante condição na fisiopatologia da DGHNA. Nosso estudo não encontrou uma diferença nas proporções quanto aos graus de DGHNA, o que pode indicar que a obesidade não se concentra em algum grau específico, mas distribui-se aos três espectros de gravidade. Ressaltase que uma amostra maior é necessária para aceitar ou refutar nossa hipótese.

Segundo Kuzuminova (2016) [18] a hipertensão arterial foi encontrada em mais de 50\% dos pacientes com DHGNA, na ausência de outros fatores de risco. Outro estudo relatou que 64\% dos pacientes portadores de DHGNA eram hipertensos [19]. Em nosso estudo, a HAS foi verificada em $80 \%$ dos pacientes, embora possa divergir dos estudos citados, a tendência a hipertensão é compartilhada pelos ensaios citados. Não constatamos uma relação associativa das médias dos níveis pressóricos, tampouco nas proporções quanto aos graus de esteatose hepática. Isso pode ser devido o tratamento pressórico dos pacientes com medicamentos anti-hipertensivos que pode ter "normalizado" os níveis no momento da aferição da pressão arterial.

A doença hepática gordurosa não alcoólica é mais prevalente em coortes de pacientes com condições metabólicas pré-existentes do que na população em geral. Especificamente, diabetes mellitus tipo II e DHGNA têm uma relação particularmente próxima. Encontrou-se associação da glicemia de jejum com os graus de esteatose, sugerindo que a acumulação esteatogênica tem uma resposta glicêmica anormal (hiperglicemia) como encalce. Como a resistência à insulina é considerado o "gatilho" no mecanismo fisiopatológico da DHGNA, podese considerar que quanto mais "disglicêmico", pior o prognóstico hepático do paciente, uma vez que reflete maior acometimento. A ligação do DM2 ou tolerância a glicose diminuída e esteatose hepática é vista em alguns ensaios clínicos e epidemiológicos. Um trabalho mostrou uma prevalência de diabetes em $70 \%$ dos indivíduos com DHGNA ${ }^{14}$. A prevalência de DHGNA entre indivíduos com DM2 no Brasil, Malásia e China foi estimada em 42\%, 49,6\% e 61\%, respectivamente $[20,21,22]$. Por outro lado, duas revisões mostraram prevalências globais menores de DHGNA em sujeitos com RI ou diabéticos. A primeira ${ }^{6}$ encontrou de 30-50\% de DM na população e a segunda ${ }^{7}(22,51 \%)$. É importante mencionar, que nesse estudo não foi verificado a presença de resistência à insulina, o que poderia elevar consideravelmente esse percentual.

Quanto as anormalidades metabólicas primárias que levam ao acúmulo de lipídios, estas não são bem compreendidas, mas podem consistir em alterações nas vias de absorção, síntese, degradação ou secreção no metabolismo lipídico hepático resultante da resistência à insulina, sendo a resistência à insulina o fator mais reprodutível no desenvolvimento de DHGNA [23].

Os estudos mostram uma faixa variável de dislipidemia na população, em números que oscilam de $20 \%$ a $90 \%$ nas populações com fígado gorduroso não alcoólico [24]. A presença de $50 \%$ dos pacientes com dislipidemia foi vista também em um trabalho indiano [24]. Outro trabalho com 304 pacientes diagnosticados com DHGNA observaram que 64\% possuíam hipertrigliceridemia, e $30 \%$ a $42 \%$ dos casos apresentaram níveis de colesterol HDL diminuídos 
[25]. Logo, e embora não tenhamos verificado associação do HDL, LDL e TG quanto a gravidade da doença, ainda podemos afirmar um caráter dislipidêmico nessa população pelas prevalências importantes. Quanto ao colesterol total sendo mais elevado nos graus inicias da doença, aceita-se que o uso de drogas hipolemiantes no estágio avançado pode ser responsável por essa inflexão. Outrossim, levantamos a hipótese de um possível mecanismo adaptativo no metabolismo lipídico a longo prazo, hipótese que necessita de novos ensaios clínicos a fim de confirmação.

No mesmo contexto, a maioria dos pacientes com DHGNA é assintomática e tipicamente ocorrem com elevação moderada nas transaminases (TGO ou TGP) [24]. Nesse trabalho, níveis normais de enzimas hepáticas foram demonstrados independente do espectro da DHGNA. Ressalta-se, que a faixa atual "normal" de transaminases hepáticas presentes em pacientes com DHGNA pode, portanto, subestimar a presença da doença [26]. Nosso trabalho não verificou associação dessas enzimas com a gravidade do infiltrado gorduroso no fígado. De modo semelhante, a fosfatase alcalina e a gama glutamil transferase também não se associaram a DHGNA.

\section{CONCLUSÃO}

A dimensão desse estudo trouxe algumas confirmações esperadas, a primeira que inúmeras manifestações clínicas acham-se no escopo da DHGNA. Nesse estudo, a hipertensão arterial, obesidade e síndrome metabólica foram as mais importantes. Segundo, embora a prevalência de Diabetes Mellitus Tipo 2 tenha sido a menor, um descontrole glicêmico tanto quanto maior aumenta com a gravidade da doença e, portanto, piora o prognóstico, razão de identificar precocemente alterações glicêmicas e a resistência à insulina. Além disso, não foram observados associação dos graus de esteatose hepática com as manifestações clínicas, o que o implica inferir que o grau independe no aparecimento das manifestações clínicas.

Entende-se, portanto, que a magnitude da DHGNA enquanto problema de saúde pública é alarmante, uma vez que sua associação com outras doenças amplia e amplifica as condições de morbimortalidade da população, o que gera a evidência e a necessidade de ações multidisciplinares de prevenção e promoção da saúde, principalmente no que tange a mudanças no estilo de vida, como alimentação e programas de atividade física.

\section{AGRADECIMENTOS}

A Secretária Estadual de Saúde de Pernambuco pelo apoio financeiro.

\section{REFERÊNCIAS BIBLIOGRÁFICAS}

1. Khoonsari M et al. Clinical Manifestations and Diagnosis of Nonalcoholic Fatty Liver Disease. Iranian J Pathol. 2017;12(2):99-105.

2. Petta $\mathrm{S}$ et al. Pathophysiology of Non-Alcoholic Fatty Liver Disease. International Journal of Molecular Sciences. 2016;17(12):2082-2107, doi:10.3390/ijms17122082

3. Italian Association for the Study of the Liver (AISF). AISF position paper on nonalcoholic fatty liver disease (NAFLD): Updates and future directions. Digestive and Liver Disease. 2017;49(5):471-483.

4. Bahrami H. Nonalcoholic Fatty Liver Disease in Developing Countries. World J Gastroenterol. 2005;11(24):3808-3809

5. Basaranoglu M, Neuschwander-Tetri BA. Doença hepática gordurosa não alcoólica: características clínicas e patogênese. Gastroenterologia e Hepatologia. 2006;2(4):282-291.

6. Bellentani S. The epidemiology of non-alcoholic fatty liver disease. Liver International. 2017;37(1):81-84, doi: 10.1111/liv.13299

7. Younossi ZM et al. Global epidemiology of nonalcoholic fatty liver disease-Meta-analytic assessment of prevalence, incidence, and outcomes. J Hepatol. 2016;64(1):73-84, doi:10.1002/hep.28431

8. Akala FA, El-Saharty S. Public-health challenges in the Middle East and North Africa. Lanceta. 2006;367(9515):961-964, doi:10.1016/S0140-6736(06)68402-X 
9. Sociedade Brasileira de Hipertensão Arterial, Sociedade Brasileira de Cardiologia, Sociedade Brasileira de Nefrologia. VI Diretrizes Brasileiras de Hipertensão Arterial. Rev Bras Hipertens. 2010;13(1):1-68.

10. World Health Organization. Obesity: preventing and managing the global epidemic. Report of a World Health Organization Consultation. Geneva: World Health Organization; 2000. p. 256. WHO Obesity Technical Report Series, n. 284.

11. OPAS. Organização Pan-Americana. XXXVI Reunión del Comitê Asesor de Ivestigaciones en Salud - Encuestra Multicentrica - Salud Beinestar y Envejecimeiento (SABE) en America Latina e el Caribe - Informe preliminar; 2002.

12. Almeda-Valdés P, Cuevas-Ramos D, Aguilar-Salinas CA. Metabolic syndrome and non-alcoholic fatty liver disease. Ann Hepatol. 2009;8(1):18-24.

13. Hamaguchi $\mathrm{M}$ et al. The metabolic syndrome as a predictor of nonalcoholic fatty liver disease. Ann Intern Med. 2005;143(10):722-728.

14. Streba LAM, Vere CC, Rogoveanu I, Streba CT. Nonalcoholic fatty liver disease, metabolic risk factors, and hepatocellular carcinoma: an open question. World J Gastroenter. 2017;21(14):4103-4110, doi:10.3748/wjg.v21.i14.4103

15. Josilda FC et al. Relação entre a esteatose hepática não alcoólica e as alterações dos componentes da síndrome metabólica e resistência à insulina. Rev Soc Bras Clin Med. 2016;14(2):79-83.

16. Fakhoury-Sayegh $\mathrm{N}$ et al. Nutritional profile and dietary patterns of lebanese non-alcoholic fatty liver disease patients: a case-control study. Nutrients. 2017;9(11):1245, doi:10.3390/nu9111245

17. Lee SW et al. The association of non-alcoholic fatty liver disease with body mass index and waist circumference in a Chinese population. J Advanc Nutrit Human Metabol. 2016;2:1-7, doi:10.14800/janhm.1483

18. Kuzminova NV et al. Arterial hypertension, obesity and non-alcoholic fatty liver disease: is there any connection? Arterial Hypertension. 2016;20(4):216-227, doi:10.5603/AH.2016.0025

19. Cotrim HP et al. Nonalcoholic fatty liver disease in Brazil, clinical and histological profile. Ann Hepatol. 2011;10(1):33-37.

20. Chan WK et al. Non-alcoholic fatty liver disease in diabetics - prevalence and predictive factors in a multiracial hospital clinic population in Malaysia. J Gastroenterol Hepatol.2013;28(8):1375-1383, doi:10.1111/jgh.12204

21. Lv WS et al. Nonalcoholic fatty liver disease and microvascular complications in type 2 diabetes. World J Gastroenterol. 2013;20(19):3134-3142, doi:10.3748/wjg.v19.i20.3134

22. Ferreira VS et al. Frequency and risk factors associated with non-alcoholic fatty liver disease in patients with type 2 diabetes mellitus. Arq Bras Endocrinol Metabol.2010; 54(4):362-368, doi:10.1590/S000427302010000400004

23. Cuenza LR et al. Correlation between severity of ultrasonographic nonalcoholic fatty liver disease and cardiometabolic risk among Filipino wellness patients. Journal of Cardiovascular and Thoracic Research. 2017;9(2):85-89, doi:10.15171/jcvtr.2017.14

24. Shil BC, Saha M, Ahmed F, Dhar SC. Nonalcoholic fatty liver disease: study of demographic and predictive factors. Euroasian Journal of Hepato-Gastroenterology. 2015;5(1):4-6, doi:10.5005/jpjournals-10018-1119

25. Marchesini G, et al. Nonalcoholic fatty liver, steatohepatitis, and the metabolic syndrome. Hepatology. 2003;37(4):917-23, doi:10.1053/jhep.2003.50161

26. Sanyal D et al. Profile of hepatic enzymes in non-alcoholic fatty liver disease in patients with impaired glucose tolerance and newly detected untreated type 2 diabetes. Indian Journal of Endocrinology and Metabolism. 2015;19(5):597-601, doi:10.4103/2230-8210.163172 